$15^{\text {th }}$ International Conference on

AEROSPACE SCIENCES \& AVIATION TECHNOLOGY,

ASAT - 15 - May 28 - 30, 2013, Email: asat@mtc.edu.eg,

Military Technical College, Kobry Elkobbah, Cairo, Egypt,

Tel: +(202) $24025292-24036138$, Fax: +(202) 22621908

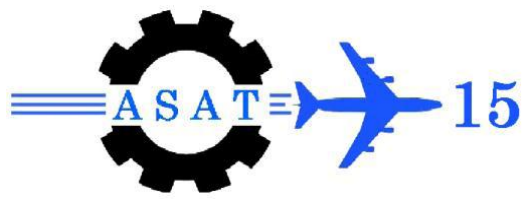

\title{
Compressive Sensing Radar Signal Processing
}

\author{
\{F. M. Ahmed, H. Kamel, K. H. Moustafa and M. Hossiny\}*
}

\begin{abstract}
This paper presents the application of Compressive Sensing (CS) theory in radar signal processing. CS uses the sparsity property to reduce the number of measurements needed for digital acquisition, which causes reduction in the size, weight, power consumption, and the cost of the CS radar receiver. Complex Approximate Message Passing (CAMP) algorithm is a fast iterative thresholding algorithm which is used to reconstruct the undersampled sparse signal and improves its Signal-to-Noise Ratio (SNR) [12- 16]. In present work, the superiority in performance of applying the CAMP algorithm in radar signal processing compared to the Digital Matched Filter (DMF), and the simple envelope detector is proved through the Receiver Operating characteristic (ROC) curves.
\end{abstract}

Keywords: Compressive Sensing, CAMP, Radar signal processing.

\section{Introduction}

In recent years, because of people's growing demand for information, the bandwidth of the signal carrying the information becomes wider. Digital signal processing technology and the rapid development of digital processing devices make digital signal processing play an important role in signal processing. Sampling is the only way to convert the analog signal into digital signal, and sampling theorem is a bridge which links between them. Sampling theorem (Shannon theorem) demands that the sampling rate should equal at least twice the bandwidth of the signal, in order to reconstruct the original analog signal without distortion.

In 2004, Donohue and Candes proposed CS theory, which is a new signal acquisition, encoding, and decoding technique [1]. CS theory combines the sampling and compression to reduce the signal sampling rate, the cost of the transmission, and the processing time. The CS theory shows that, when the signal has the characteristic of sparsity, the original signal can be exactly or approximately reconstructed from undersampled measurements.

In radar signal processing, in order to accurately probe the target, large-bandwidth signals need to be launched (very narrow pulse duration), which requires a very high sampling rate to accurately estimate the target parameters. The CS theory may be applied in radar signal processing to manage this issue [1-8].

This paper is organized as follows; after the introduction, section 2 gives a survey on the bases of CS theory. Section 3 focuses on the feature of the CAMP algorithm (kind of the iterative thresholding algorithms). Performance evaluation through the ROC curves of the CAMP algorithm compared to the DMF and the simple envelope detector with comparator are presented in section 4. Finally, conclusion comes in section 5.

\footnotetext{
*Egytian Armed Forces, Egypt.
} 


\section{Compressive Sensing theory}

Based on the characteristic of sparsity of signal, CS theory converts the high dimensional signal to a lower dimensional signal using a sensing matrix, A, then reconstructs the original signal with high probability using a small number of measurements. Considering the problem of recovering a sparse signal, $x$, from an undersampled set of measurements, $y$ :

$$
\begin{gathered}
\mathrm{y}=\mathrm{Ax}+\mathrm{n} \\
\delta=\mathrm{M} / \mathrm{N} \quad, \quad \rho=\mathrm{K} / \mathrm{M}
\end{gathered}
$$

where $y$ is $(M \times 1)$ measurement matrix, $A$ is $(M \times N)$ sensing matrix, $x$ is $(N \times 1)$ sparse radar signal, $\mathrm{n}$ is Gaussian random noise with zero mean and unity variance, $\rho$ is the radar signal sparsity, and $\delta$ is the undersampling factor. The process of compression and reconstruction of signal using CS theory is organized, as shown in figure (1) [7].

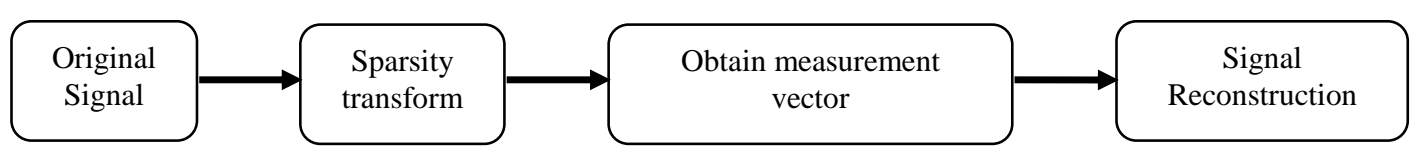

Fig. 1 General CS diagram.

As shown in figure (1), application of CS in radar signal processing may be organized separately in three aspects: sparse representation of radar signal, designing of sensing matrix, and reconstruction of the radar signal. These aspects shall be discussed in the following subsections:

\subsection{Sparse representation of radar signal}

The Fourier transform and the Wavelet transform are used to provide more direct analysis for the radar signal. The purposes of these transformations are aimed at representing the radar signal in sparse form. Suppose, s, is one-dimensional discrete-time signal with finite length and real value. From the Matrix theory, the signal, s, can be represented by its orthonormal basis [10]:

$$
\mathrm{s}=[\mathrm{s} 1 \mathrm{~s} 2 \mathrm{~s} 3 \ldots \mathrm{sN}]
$$

$$
\psi=\text { Fourier or wavelet transform }[\mathrm{s}]
$$

So, the sparse representation, $\mathrm{x}$, of the signal, $\mathrm{s}$, is given in equation (5):

$$
\mathrm{x}=\psi \mathrm{s}
$$

where, $\mathrm{x}$, is the sparse representation of the original signal, and $\psi$ is the representation in the transformation domain (Fourier / Wavelet). Suppose k be non-zero number of elements of, $\mathrm{x}$. If $\mathrm{k}$ is smaller than $\mathrm{N}$, so the signal, $\mathrm{x}$, is sparse or compressible. Sparsity reduces the number of zero coefficients which help to reduce the sampling rate with the factor $M$ [12] which is given by equation (6) :

$$
\mathrm{M} \geq \mathrm{k}^{2} \ln \mathrm{N}
$$

where $\mathrm{M}$ is the number of measurements, $\mathrm{N}$ is the number of Nyquist rate samples, and $\mathrm{k}$ is the number of the non-zero coefficients. 
From the nature of the radar signal, it is clear that the radar signal has a sparsity property. This is because the number of targets is typically much smaller than the number of resolution cells in the illuminated area or volume. This means that, the step of sparsity transformation for the received radar signal is not included in the present work. So, the compressed measurement vector may be obtained directly from the received radar signal.

\subsection{Designing the sensing matrix $A$}

The sensing matrix A which contains random numbers is designed to ensure that the sparse signal, s, can be reconstructed perfectly according to a sufficient and a necessary conditions. The sufficient condition is that the matrix $\mathrm{A}$ has the coherence property $\mu(\mathrm{A})$, which is the largest absolute inner product between any two columns ( $i, j$ ) of $A$ as in equation (7) [11]. The coherence property of the matrix $\mathrm{A}$ is used to ensure that the matrix $\mathrm{A}$ is sparse matrix by designing it to be orthonormal matrix:

$$
\mu(\mathrm{A})=\max _{1<i<j \leq \mathrm{N}} \frac{\left|\left(\mathrm{A}_{\mathrm{i}}, \mathrm{A}_{\mathrm{j}}\right)\right|}{\left\|\mathrm{A}_{\mathrm{i}}\right\|_{2} \|}
$$

The necessary condition of the matrix A is the Restricted Isometry Property (RIP), which is given in equation (8), which provides guarantees of uniqueness when the measurement vector $\mathrm{y}$ is obtained without error by determining the number of measurements $\mathrm{M}$ (equation 6). RIP solves two problems: the first problem appears when the pseudo inverse is used to reconstruct the sparse signal, s, as the matrix A isn't invertible [12]. The second problem is the two kinds of errors (radar system noise and Mean Square Error (MSE)). Under these problems, it is no longer possible to guarantee uniqueness, which is controlled by the RIP property, which insures that the recovery process is stable in presence of the noise, and to control the tolerant for both types of errors [12]:

$$
(1-\delta)\left\|_{s}\right\|_{2}^{2} \leq\|A, s\|_{2}^{2} \leq(1+\delta)\|s\|_{2}^{2}
$$

\subsection{Reconstruction of radar signal}

In the CS theory, because the number of measurements $M$ is lower than the number of the samples $\mathrm{N}$ of the original sparse radar signal, $\mathrm{s}$, the problem is solvable. It can be solved by $\ell 1$ norm minimization, and the estimated radar signal, $\hat{s}$, is given by the equation (9) [12]:

$$
\hat{s}_{(\lambda)}=\arg \min \frac{1}{2}\|\mathrm{y}-\mathrm{A} \cdot s\|+\lambda\|s\|
$$

where $^{\lambda}$ is the regularization parameter that controls the update of the measurement vector, $y$. Equation (9) is a convex function and can be solved by standard techniques such as interior point or homotopy methods. However, these approaches are computationally expensive; therefore iterative algorithms are applied with inexpensive computations like the CAMP algorithm $[15,16]$.

\section{Complex Approximate Message Passing (CAMP)}

The Complex Approximate Message Passing (CAMP) algorithm is a kind of Iterative Thresholding algorithms, which refines the reconstructed signal at each iteration by a thresholding steps. In the present work, this algorithm is used to reconstruct the radar signal, as shown in figure (2) [13-16]. 


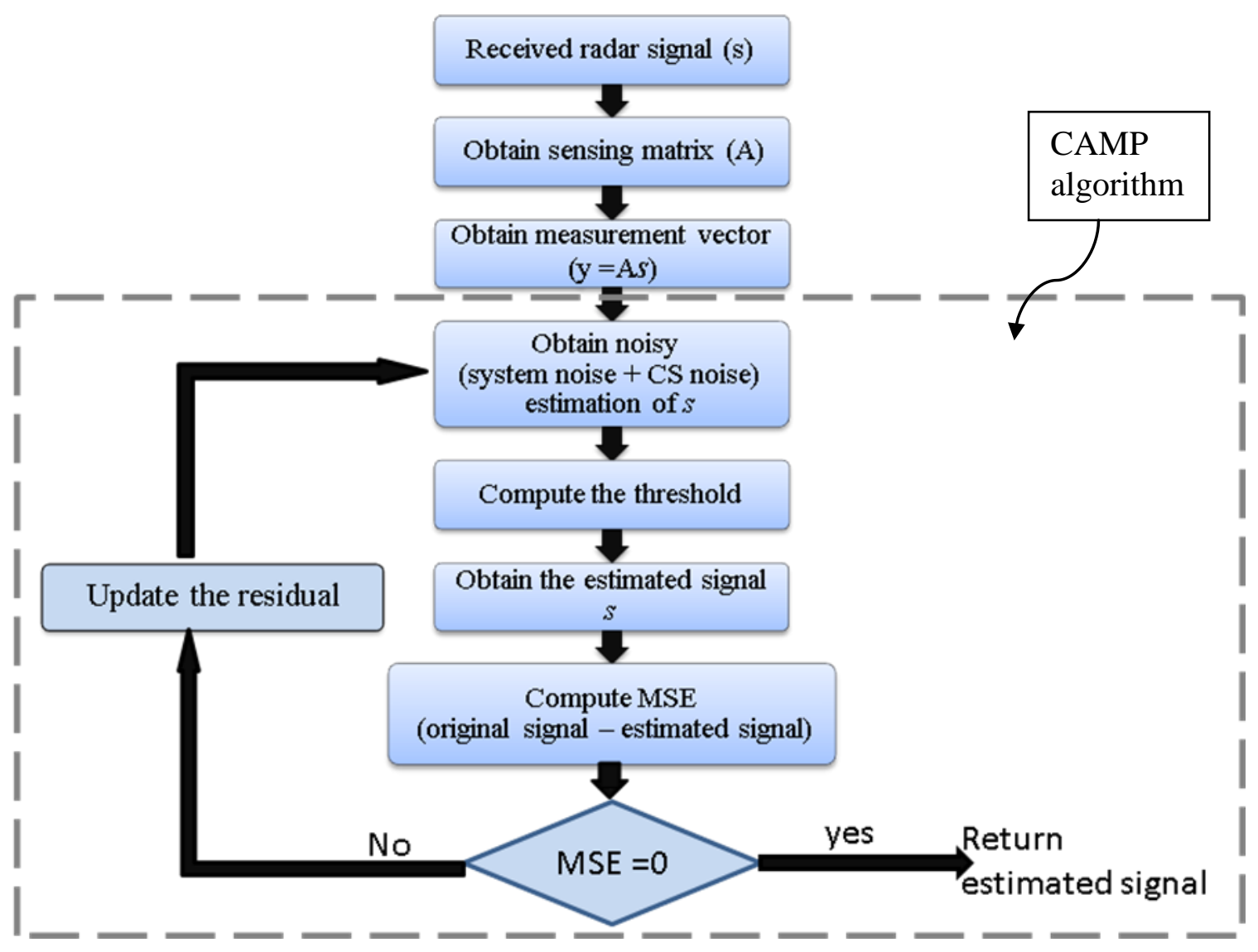

Fig. 2. Flowchart of the CAMP algorithm.

As shown in figure (2), the CAMP algorithm can be summarized as follows, where the estimated signal is firstly initialized:

$\hat{s}^{0}=0, \quad$ zo $=\mathrm{y}$

where $\hat{s}^{0}$ is the estimated value of the signal, s, and zo is the measurement matrix at a certain iteration. Then CAMP algorithm firstly compute the noisy estimation of the radar signal, then the threshold value is determined from this noisy estimation.

In the previous work [16], this threshold was chosen according to sorting the noisy estimation of the radar signal, $\tilde{s}$, and choosing the value number $(\mathrm{M})$, by performing different simulation trails, it is found that setting this threshold value as the average of the noisy estimation of the radar signal, $\tilde{s}$, gives better results (equation 11 ).

$$
\begin{gathered}
\tilde{s}^{\mathrm{t}}=\mathrm{A}^{\mathrm{t}} \cdot z^{\mathrm{t}-1}+\hat{s}^{\mathrm{t}-1} \\
\tau=\operatorname{average}\left(\tilde{s}^{\mathrm{t}}\right)
\end{gathered}
$$

where $\tilde{\mathrm{x}}^{\mathrm{t}}$ is the non sparse noisy estimation of the signal, s, and ${ }^{\tau}$ is the threshold of the noisy estimated non sparse signal. 
Then the measurements will be updated to be prepared to the next iteration for reconstructing the sparse radar signal, s, by using the iteration soft threshold function $\eta$ :

$$
\begin{gathered}
\hat{\mathrm{x}}^{\mathrm{t}}=\eta\left(\tilde{\mathrm{x}}^{\mathrm{t}} ; \tau\right) \\
\mathrm{z}^{\mathrm{t}}=\mathrm{y}-\mathrm{A} \cdot \hat{\mathrm{x}}^{\mathrm{t}-1}+\mathrm{z}^{\mathrm{t}-1} \cdot \operatorname{MSE}
\end{gathered}
$$

where $\tau$ is the threshold value, and MSE is the Mean Square Error (the error due to the mismatches between the original and the reconstructed radar signal). The previous algorithm is repeated many times in order to minimize the MSE, which is the difference between the original and the reconstructed signal. When the MSE value becomes less than the tolerance value, the iteration is stopped.

To evaluate the performance of the CAMP algorithm, it is compared to the DMF as a traditional signal processing technique as well as the simple envelope detector through the ROC.

\section{Simulation Results}

Considering an analog received pulse radar signal with duration $\delta \mathrm{t}=4 \mu \mathrm{s}$. According to Shannon theory, the sampling rate is chosen to be $1 \mathrm{MHz}$, so the radar signal has four samples in its pulse duration. The simulation results are obtained for three schemes: the CAMP algorithm, the envelope detector with comparator, and the DMF.

\subsection{The CAMP algorithm}

Firstly, the received radar signal is considered to be a perfect radar signal which doesn't have any noise or clutter as shown in figure (3), consider a sparse radar signal of length 100 samples, which can be reconstructed from a minimum number of measurements $\mathrm{M}=75$ samples according to equation (6). The signal is considered to have four peaks, so the number of non zero coefficients is $\mathrm{k}=4$ (sample at the pulse width), and the signal sparsity $\rho=\mathrm{K} / \mathrm{M}=0.053$ and undersampling factor $\delta=\mathrm{M} / \mathrm{N}=0.75$. The spectrum of both signals are the same, so the reconstructed radar signal by the CAMP algorithm is completely like the original radar signal.

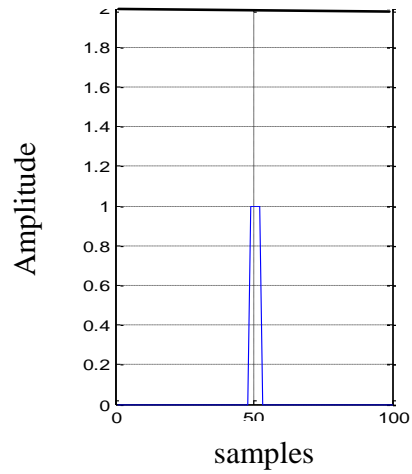

(a)

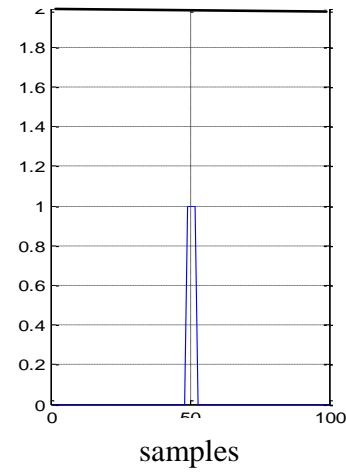

(b)

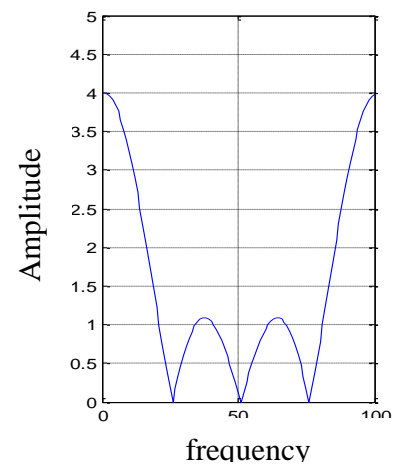

(c)

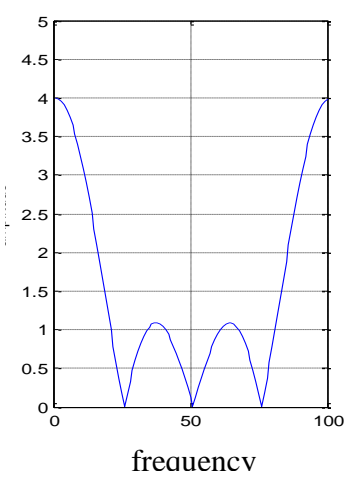

(d)

Fig. 3 Reconstruction of the noise free radar signal by using the CAMP algorithm (a) original signal, (b) reconstructed signal (c) spectrum of original signal,

(d) spectrum reconstructed signal. 
For a case of noisy radar signal the reconstructed signal will be like the original signal but without noise, and the spectrum of the reconstructed signal will be as the ideal noise free signal, as shown in figure (4).

The Mean Square Error (MSE) is the square of difference between the original radar signal and the reconstructed signal, and it accurately predicts the behavior of the CAMP algorithm. It changes from iteration to iteration, and it depends on the sparsity $\rho$ and undersampling factor $\delta[15]$, as shown in figure (5).

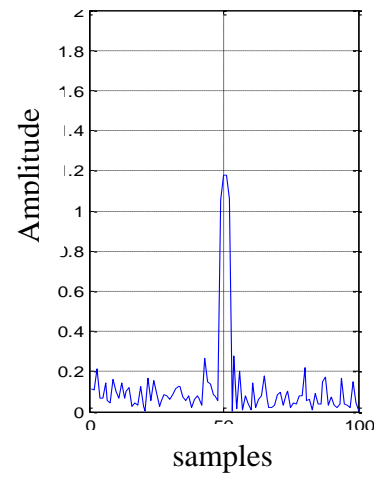

(b)

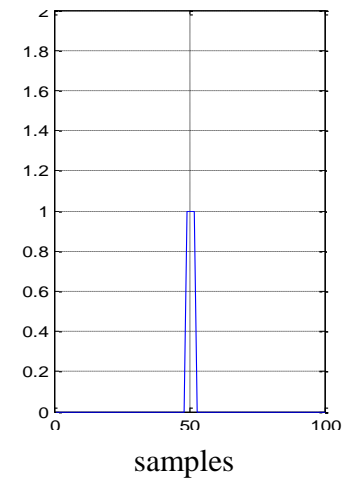

(c)
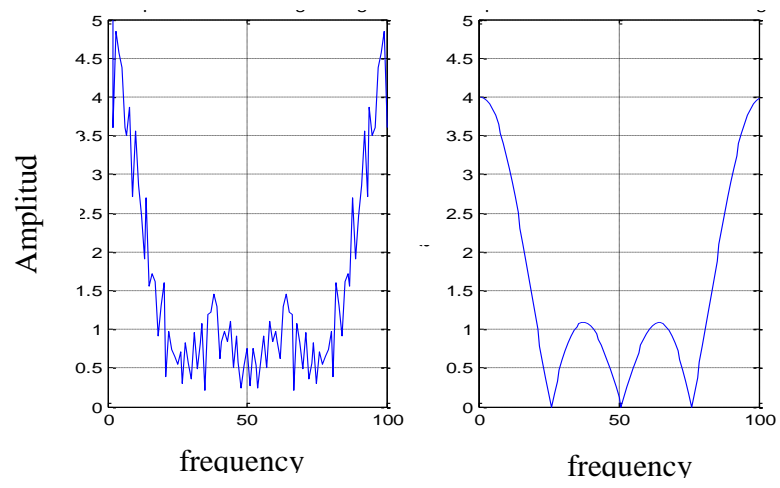

(d)

Fig. 4 Reconstruction of the noisy radar signal by using the CAMP algorithm at Pfa = 10-5: (a) original signal, (b) reconstructed signal, (c) spectrum of original signal, (d) spectrum of the reconstructed signal

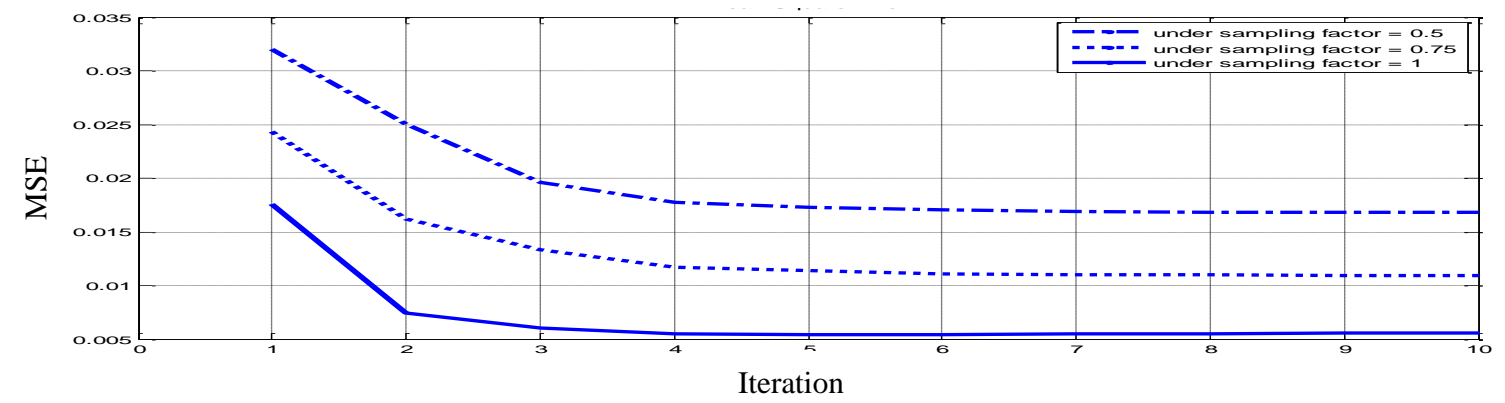

Fig. 5 The MSE at different values of the undersampled factor $\delta$ at $\mathrm{Pfa}=10-5$.

Figure (5) shows that the MSE of the reconstructed radar signal by using the CAMP algorithm approximately tends to zero after four iterations. The undersampled factor effects the MSE, where as the undersampled factor increases (number of measurements increase) the MSE decreases.

The undersampled factor (number of measurements) affects the probability of detection of the CAMP algorithm, where as the undersampled factor increases (number of measurements increases), the probability of detection of the reconstructed radar signal will increase [16], as shown in figure (6). 


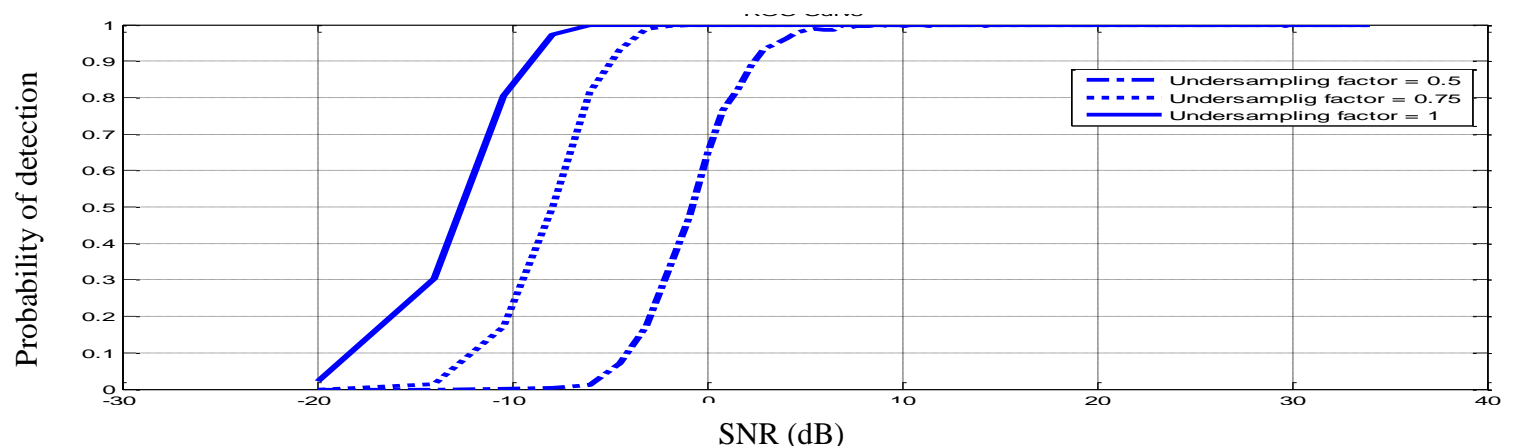

Fig. 6 ROC of the CAMP algorithm at different values of the undersampled factor $\delta$ at Pfa $=\mathbf{1 0 - 5}$.

\subsection{The envelope detector}

The envelope detector is used by a radar system when the phase of the received pulse is unknown (non-coherent), and it is used with comparator to detect the received radar signal (target), as shown in figure (7).

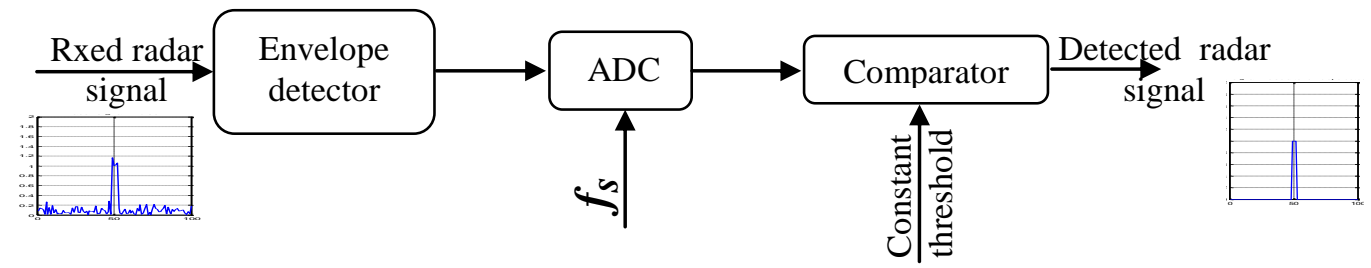

Fig. 7 Simple block diagram of the envelop detector with comparator.

The envelop detector detects the received radar signal peaks by comparing it to a pre-determinable threshold, which achieve a predesigned probability of false alarm, as shown in figure (8).

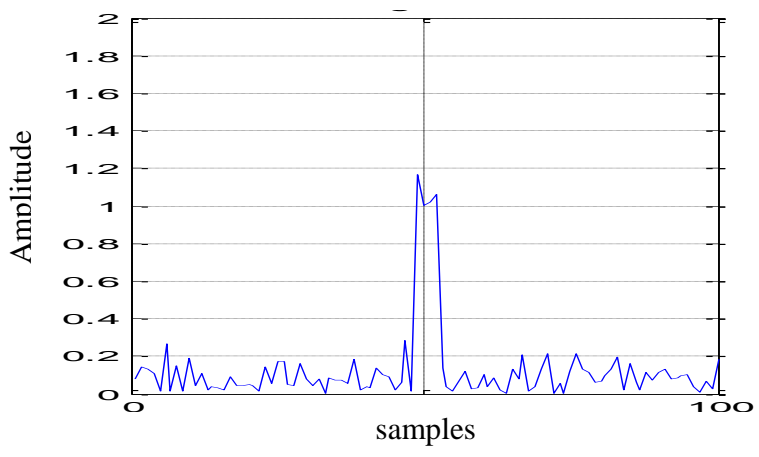

(a)

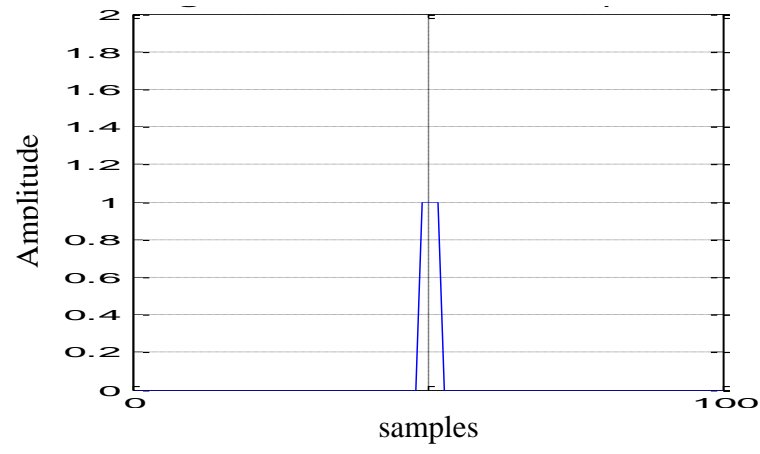

(b)

Fig. 8 The effect of the envelope detector on the noisy radar signal at $\mathrm{Pfa}=10-5$ : radar signal, (b) radar signal after the envelop detector and comparator. 


\subsection{Digital Matched Filter}

The DMF is used to maximize the SNR of the radar signal [16], and it is commonly used in radar applications, in which a known signal is reflected, and examined for common elements of the transmitted signal. Figure (9) shows a realization of the sub-pulse DMF for a rectangle video pulse [17].

In the present work the radar signal has four samples in its peak, so the DMF will have four delay blocks in its design. The outputs of every delay block will be collected together by using a summator, which amplifies the amplitude of the radar signal, as shown in figures (9), (10).

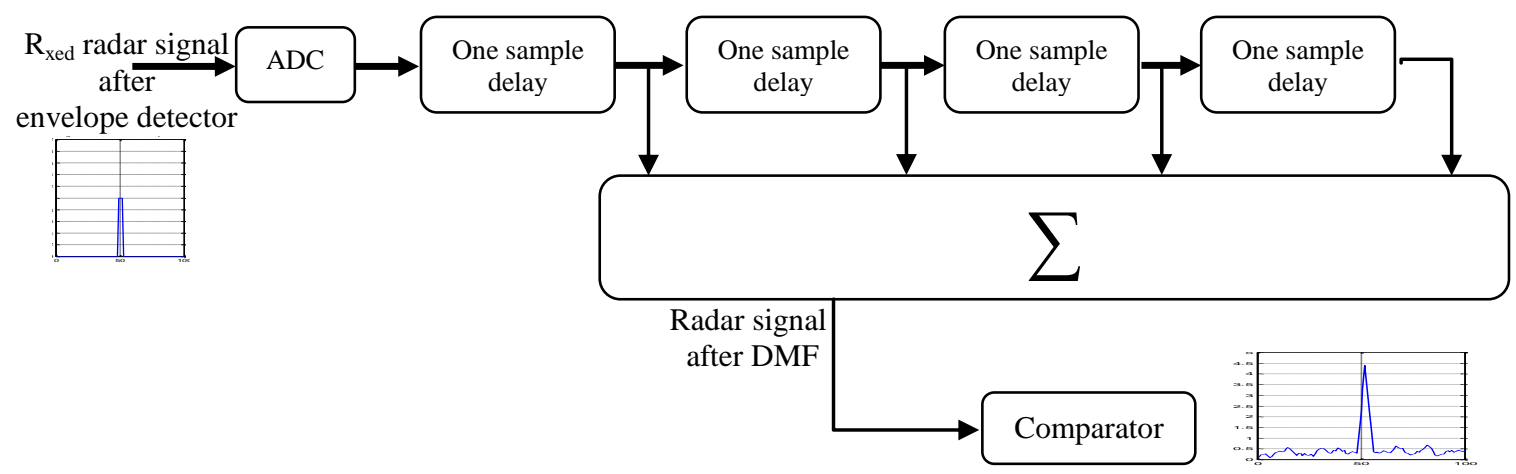

Fig. 9 Simple block diagram of the DMF.

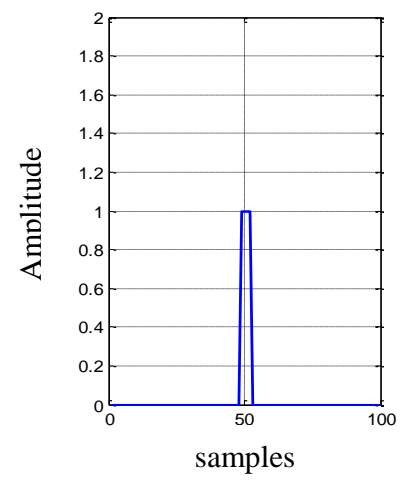

(a)

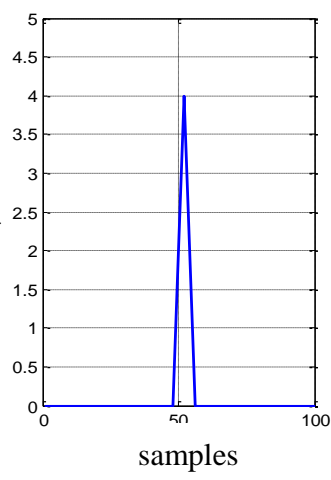

(b)

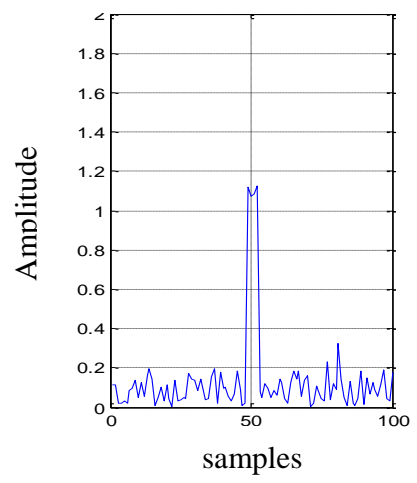

(c)

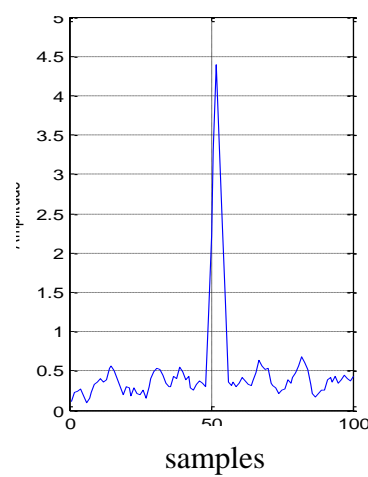

(d)

Fig. 10 The effect of the DMF on the received radar signal:

(a) noise free signal, (b) noise free signal after DMF, (c) noisy signal, (d) noisy signal after DMF.

The ROC curves are plotted to compare between the performance of the envelope detector, the CAMP, and the DMF, at constant probabilities of false alarms of 10-4, 10-5, and 10-6, as shown in figures (11), (12), and (13) respectively.

The constant probability of false alarm is controlled by the threshold. The threshold in the simple envelop detector, and the DMF is a constant threshold, which is set after the envelope detector and after the DMF, but the threshold of the CAMP algorithm is set inside the algorithm itself (soft thresholding function). 
The superiority of the CAMP algorithm in detecting the radar signal compared to the DMF or the simple envelope detector is very clear from figures (11), (12), and (13). Regarding to the complexity of real time computation, it is clear that the CAMP algorithm needs more calculations than the DMF or the envelope detector.

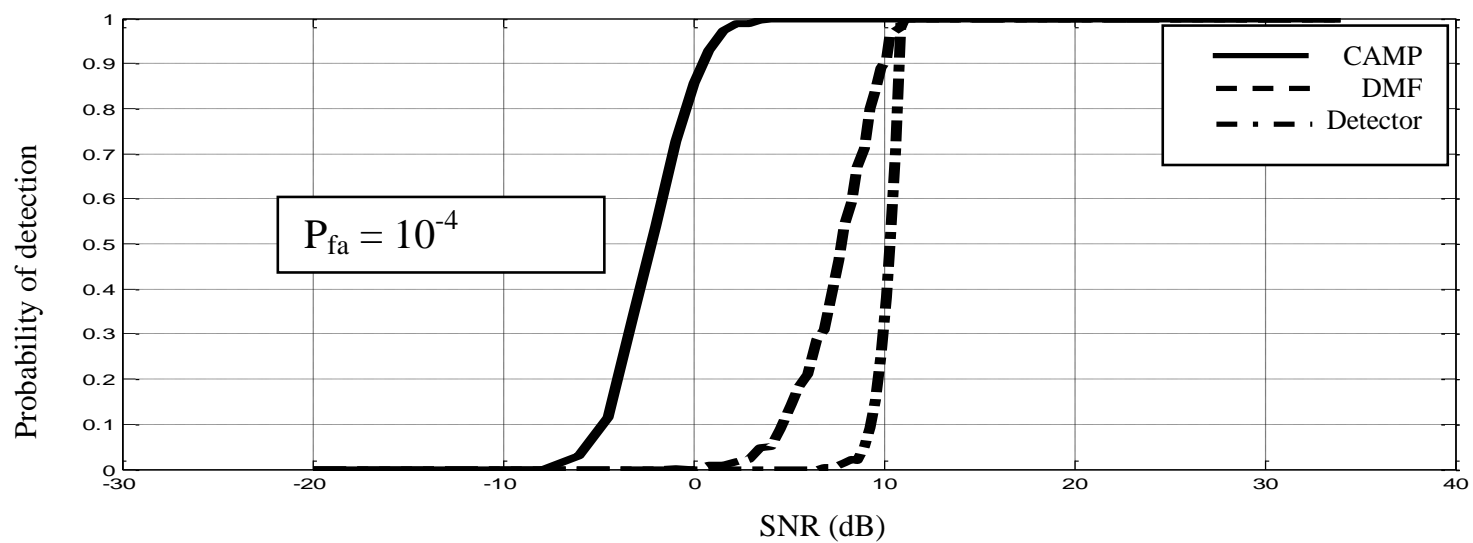

Fig. 11 ROC of the CAMP algorithm, the DMF, and the simple envelope detector at Pfa $=10-4$.

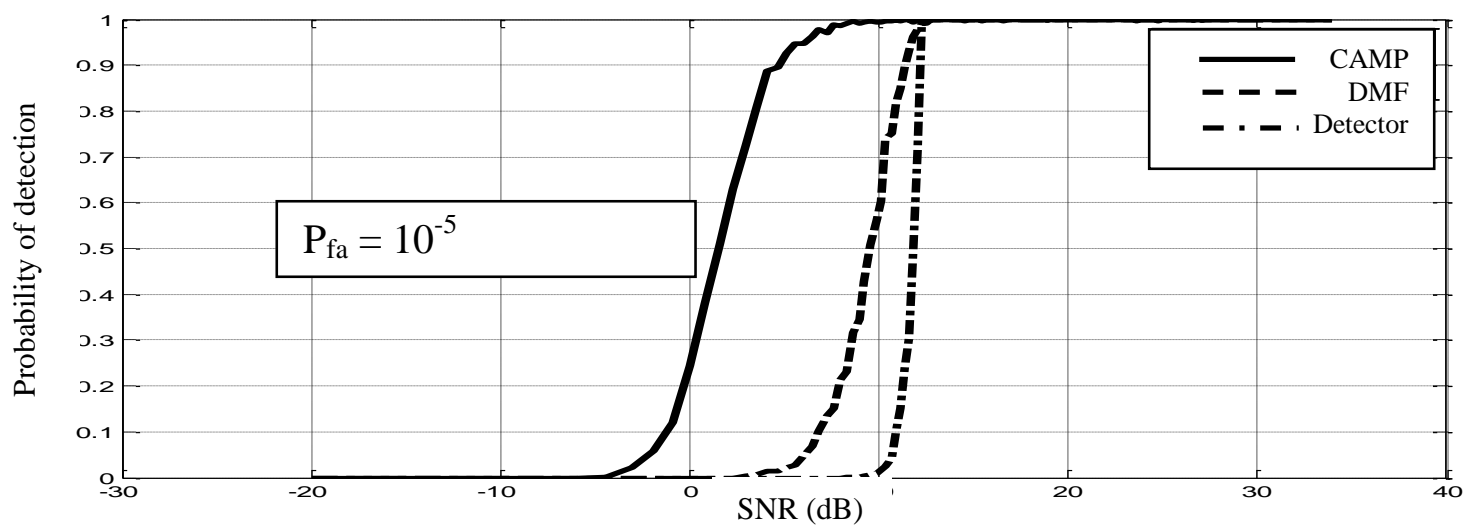

Fig. 12 ROC of the CAMP algorithm, the DMF, and the simple envelope detector at Pfa $=10-5$.

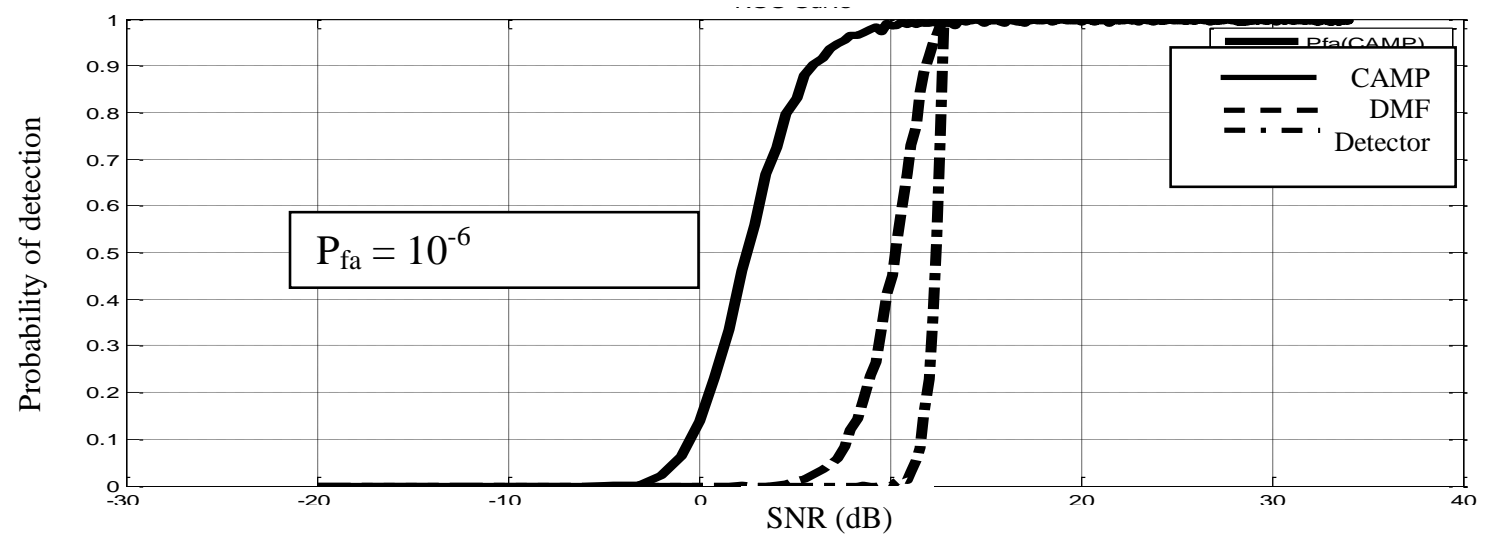

Fig. 13 ROC of the CAMP algorithm, the DMF, and the simple envelope detector at Pfa $=$ 10-6. 


\section{Conclusion}

This paper gives a general description about CS theory, the sparsity property of the radar signal, and focuses on the reconstruction of the radar signal from undersampled measurements by using the CAMP algorithm. A suggested method based on simulation is proposed for calculating the threshold value according to the average of the noisy estimation of the signal in the CAMP algorithm, which gives better results. Increasing the number of measurements reduce the MSE and increases the probability of detection of the reconstructed radar signal by using the CAMP algorithm. The detection performance of applying the CAMP algorithm on the pulse radar signal was found to be better than that of the DMF and envelope detector. The CAMP algorithm can be used in the radar signal processing to improve the SNR better than the DMF, and the envelope detector. On the other hand, complexity and time of calculation may be increased, and must be studied well for the real time implementation.

\section{References:}

[1]. D. L. Donoho, "Compressed sensing", IEEE Trans, Inf. Theory, Apr. 2006.

[2]. R. G. Baraniuk, and T. P. H. Steeghs, "Compressive radar imaging", In Proc. IEEE Radar Conf, 2007.

[3]. M. A. Herman, and T. Strohmer, "High-resolution radar via compressed sensing", IEEE Trans. Signal Process, 2009.

[4]. Y. Wang, and G. Leus, "Direction estimation using compressive sampling array processing", Signal Process, 2009.

[5]. J. H. G. Ender, "On compressive sensing applied to radar", Signal Process, May 2010.

[6]. L. Anitori, M. Otten, and P. Hoogeboom. "Compressive sensing for high resolution radar imaging", 2010.

[7]. S. Shah, Y. Yu, "Step-frequency radar with compressive sampling SFR-CS", Signal Process, (ICASSP), 2010.

[8]. L. C. Potter, E. Ertin, J. T. Parker, "Sparsity and compressed sensing in radar imaging", Proc. IEEE, Jun. 2010.

[9]. Zhu Lei, "Application of Compressed Sensing Theory to Radar Signal Processing", Speech, and Signal Process, 2010.

[10]. C. Hegde and R. G. Baraniuk, "Sampling and recovery of a sparse sum of pulses, 2010.

[11]. Arian Maleki, "Approximate Message Passing Algorithms for Compressed Sensing", September 2011.

[12]. D. L. Donoho, A. Maleki, and A. Montanari. "Message passing algorithms for compressed sensing", 2009.

[13]. D. L. Donoho, A. Maleki, and A. Montanari. "Construction of message passing algorithms for compressed sensing", 2010.

[14]. A. Maleki, L. Anitori, "Asymptotic analysis of complex LASSO via complex approximate message passing (CAMP)", 2011.

[15]. M. Fornasier and H. Rauhut, "Iterative thresholding algorithms applied and Computational Harmonic Analysis", 2008.

[16]. Laura Anitori, and P. Hoogeboom, "Compressive CFAR Radar Detection". Conf. Acoust., radar, and Signal Process, 2012.

[17]. Gaspare Galati, "Advanced radar techniques and systems", 1988. 